\title{
Primary Pyomyositis: A rare cause of Painful leg in a diabetics?
}

\author{
Tanveer H. Banday ${ }^{1}$, Sadaf Bashir bhat ${ }^{2}$, Shah Naveed ${ }^{3}$, Irfan Wani ${ }^{4}$, \\ Danchand ${ }^{5}$, Sabreen bashir bhat, \\ Assistant professor, Department of medicine, AIMS Bangalore.
}

\begin{abstract}
Pyomyositis an acute/subacute, destructive, and suppurative bacterial infection of skeletal muscle. Pyomyositis is not secondary to a contiguous infection from other areas, such as the skin, bone, or soft tissue. Common intropical climates, pyomyositis accounts for four percent of all surgical admissions in some tropical countries.

Most patients with this process have underlying immune compromised like(diabetes, HIVetc), but may also affects healthy young individuals [1,2]. Most patients present with tender indurated muscle swelling that ultimately progresses to involve the overlying tissue. Muscles of the pelvic area and lower extremities are most commonly affected. An abscess of the thigh, quadriceps, and iliopsoas muscles are relatively common, but both the upperand lower body may be affected [3].

A delay in diagnosis may result in compartmentsyndrome, sepsis, and death. The long-term sequelae of pyomyositis includeosteomyelitis of adjacent bones, myonecrosis ,muscle scarring, prolonged hospitalization and significant functional impairment [4].
\end{abstract}

Keywords; pyomyositis, diabeties, leg pain.

\section{Case report}

We report a patient with type II diabetes 10years in duration who presented with a painful leg.He hadhistory of increasing pain in right thigh posterior aspect .He was unable to weight-bear and had systemic features of malaise, fever, and rigors. There was no history of trauma.

On examination patient was febrile, pulse 120beats/min bloodpressure $120 / 76 \mathrm{mmHg}$ respiratory rate of 16breaths /min.Local examination showed ;On Inspection -atense, and swollen right thigh .On palpation tender and indurated. There was no erythema or lymphadenopathy present. Swelling progressed over next 2 days and involved whole right lower limb just above ankle joint and proximally upto right inguinal ligament and flanks. The overlying skin was indurated. Peripheral pulsation ,neurological examination was normal.

Investigation;Laboratory results showed a total leucocyte count $\left(10500 / \mathrm{mm}^{3}\right)$ differential leucocyte count, neutrophilia (78\%) with left shift, C-reactive protein _250 mg/l, and creatinine kinase 456 units/l. Blood glucose was $480 \mathrm{mg} / \mathrm{dl}$ random on day 1 of admission which was controlled within range of 110 to $140 \mathrm{mg} / \mathrm{dl}$.Serum urea $104 \mathrm{mg} / \mathrm{dl}$, serum creatinine $3.3 \mathrm{mg} / \mathrm{dl}$, SGOT $32 \mathrm{U} / \mathrm{L}$, SGPT 63/dl. X RAY knee and thigh was normal on day of admission. HbsAg, anti HCV and HIV serology was nonreactive. Two sets of blood cultures grew MSSA within $48 \mathrm{~h}$. There was no suggestion of deep venous thrombosis on $2 \mathrm{D}$ color Doppler. Magnetic resonance imaging of right limb showed myofascitis in posterior and medial compartment of thigh with areas of necrosis with associated cellulitis(fig1)

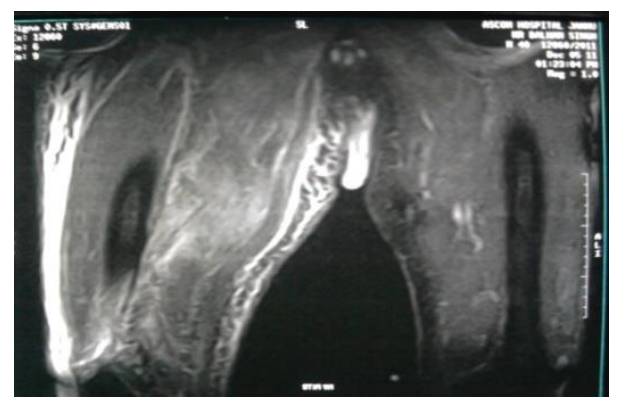

Computerized tomography (CT) abdomen showed right lateral and posterior abdominal wall myofascitis and cellulitis with extension into right hip region and left lateral abdominal wall, bilateral pleural effusion with right side basal atelectasis(fig2) 


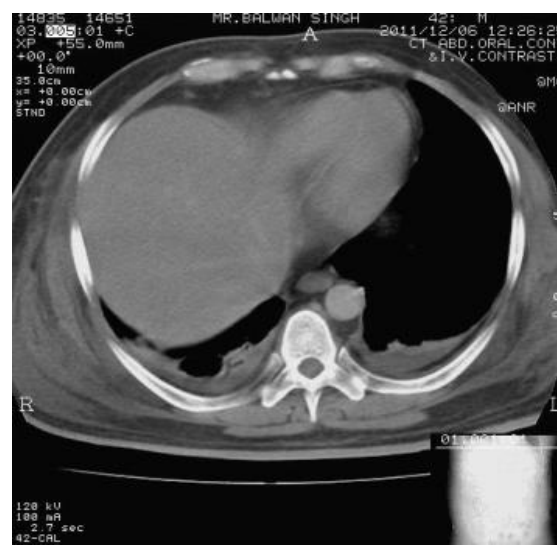

The patient was treated withantibiotics( pippercillin tazobactum, vancomycin, clindamycin) and insulin infusion for glycemic control. The tissue samples grew MSSAPseudomonas, leading to a change in antibiotics to vancomycin and ciprofloxacin. He had a prolonged stay in hospital but patient was managed conservatively mostly with parental antibiotics without requiring much surgical intervention and was discharged from hospital without any complication.

\section{Discussion}

Pyomyositis is still probably a rare process, while transient, self-limited, vague, unexplained hip, back, and leg pain are omnipresent.Although perfectly healthy individuals can suffer from spontaneous pyomyositis, this is still generally a disease of the immunocompromised .Pyomyositis, a primary muscle infection, is probably the result of a transient bacteremia. Characteristically, muscles of the lower body are most commonly affected, such as the quadriceps, gluteal, and iliopsoas muscles. The most common organism is S. aureus, present in more than $75 \%$ of the cases.

Diabetes is an important contributing factor to pyomyositis by predisposing the skeletal muscle to damage [5,6])and increasing susceptibility to infections. Review of the pyomyositis literature suggests that its incidence in diabetes has increased from 8\% of cases in 1971-1991 to 31\% of cases in recent reports [7].

The pathogenesis of pyomyositis is confusing because intact muscle is usually quite resistant to infection. Curiously, many patients relate trauma, seemingly minor in nature. It is generally considered secondary to a bacteremia. No characteristic source or portal of entry has been identified. The pathogenesis of pyomyositis is multifactorial. Transient bacteremia with concomitant muscle damage may be a causative factor.

Rarer infectious agents include Streptococcuspyogenes, Streptococcus pneumoniae, Escherichia coli, Mycobacteriumavium, and gram-negative bacteria [4].Pyomyositis progresses through three clinical stages [8]. The initial invasivestage begins with cramping and aches and a low-grade feverStage 2, muscle abscesses have formed and local and systemic manifestationsare present. Stage3, which is characterized by signs of toxicity and septic shock.

Routine laboratory investigations are rarely helpful. Inflammatory markers such as C-reactive protein may be elevated [3], and 50\% of patients have a leucocytosiswith a left shift [9]. Muscle enzyme levels are variable. Blood culturesare positive in 5-31\% of cases [9]. Plain radiography is often unremarkable andmainly used to rule out primary bone lesions. MRI is the imaging modality ofchoice, especially in the initial stages. It demonstrates diffuse muscle inflammationand abscess formation. Ultrasonography and CT are useful in localizingintramuscular abscesses and guiding needle.

The mainstay of treatment is drainage of the abscess, coupled with prolonged antibiotic therapy. Percutaneous needle drainage may occasionally be successful, but often these patients require deep and extensive I \& D. The majority of misdiagnosed patients are probably first treated as nonspecific myofacial pain or simple cellulitis, or other nonspecific soft tissue entities before the abscess is obvious.

The duration of antibiotic therapy can range from a few days to a more prolonged course, as occurredin this case. The diagnosis of pyomyositis requires a high index of suspicion and should be considered in all patients with fever and muscle pain. This is particularly importantin patients with diabetes. Successful treatment requires early recognition, appropriate use of antibiotic therapy,and debridement and drainage of the affected tissues.

\section{III.}

\section{Conclusion}

In conclusion we want emphasize that pyomyositis should be considered as one of differential of pain leg especially in diabetic patients and second these patients can be managed conservatively and complication could be prevented /minimized with early introduction of appropriate antibiotics. The next time you see a 
diabetic with a vague, hard-to-describe, deep aching pain in the pelvis, hips, buttocks, or thighs, give primary pyomyositis a consideration.

\section{References}

[1]. Yoneda M, Oda K: Type 2 diabetes complicated by multiple pyomyositis. InternMed 42:174-177, 2003

[2]. Rodgers WB, Yodlowski ML, Mintzer CM: Pyomyositis in patients who have the human immunodeficiency virus: case report and review of the literature. J Bone Joint Surg Am 75:588-592, 1993

[3]. Christin L, Sarosi GA: Pyomyositis in North America: case reports and review.Clin Infect Dis 15:668-677, 1992

[4]. Bickels J, Ben-Sira L, Kessler A, Wientroub S: Primary pyomyositis. J Bone JointSurg Am 84:A2277-A2286, 20026

[5]. Walling DM, Kaelin WG Jr: Pyomyositis in patients with diabetes mellitus. Rev InfectDis 13:797-802, 1991

[6]. Pozzilli P, Leslie RD: Infections and diabetes: mechanisms and prospects for prevention. Diabet Med 11:935-941, 1994

[7]. Patel SR, Olenginski TP, Perruquet JL, Harrington TM: Pyomyositis: clinical features and predisposing conditions. $J$ Rheumatol24:1734-1738, 1997

[8]. 8. Chiedozi LC: Pyomyositis: review of 205 cases in 112 patients. Am J Surg 137:255- 259, 1979

[9]. 9. Fan HC, Lo WT, Chu ML, Wang CC: Clinical characteristics of Staphylococcal pyomyositis. J MicrobiolImmunol Infect 35:121124, 2002 Diabetic pyomyositis 1744 